\title{
Wernicke's encephalopathy in a patient with non- Hodgkin's lymphoma post-Autologous HSCT
}

\author{
João Paulo de Vasconcelos Leitão ${ }^{1}$ \\ Romélia Pinheiro Gonçalves Lemes ${ }^{2}$ \\ Maritza Cavalcante Barbosa ${ }^{3}$ \\ Beatriz Stela Gomes de Souza Pitombeira Araújo ${ }^{1}$ \\ Karine Sampaio Nunes Barroso ${ }^{1}$ \\ Jacques Kaufman ${ }^{1}$ \\ Talyta Ellen de Jesus dos Santos ${ }^{3}$ \\ (D) Anna Thawanny Gadelha Moura ${ }^{3}$ \\ André Rodrigues Façanha Barreto ${ }^{4}$ \\ Fernando Barroso Duarte 5
}

1. Doctor, Residence in Hematology, Bone Marrow Transplantation Service at Walter Cantidio University Hospital - Fortaleza (CE), Brasil. 2. Ph.D. in Pharmacy, Professor of Hematology, Federal University of Ceará - Fortaleza (CE), Brasil. 3. Ph.D in Pharmaceutical Sciences, Federal University of Ceará - Fortaleza (CE), Brasil. 4. Doctor, Residency in Radiology and Diagnostic Imaging of Walter Cantídio University Hospital - Fortaleza (CE), Brasil. 5. Ph.D. in Surgery, Chief of Bone Marrow Transplantation Service at Walter Cantidio University Hospital - Fortaleza (CE), Brasil.

http://dx.doi.org/10.1590/1806-9282.64.10.882

\section{SUMMARY}

Wernick's Encephalopathy (WE) is an acute neuropsychiatric syndrome caused by thiamine deficiency post hematopoietic stem cell transplant (HSCT). WE is associated with high mortality and morbidity rates, but due to its rare occurrence, it is rarely considered in patients submitted to this procedure. Considering that, the manuscript reports the clinical characteristics and the possible factors that predisposed the occurrence of WE in a patient with non-Hodgkin's lymphoma post-Autologous HSCT. We conclude that WE should be considered in patients submitted to autologous HSCT associated with prolonged use of TPN and malnutrition.

KEYWORDS: Wernicke's encephalopathy; non-hodgkin's lymphoma; autologous hsct; thiamine deficiency.

Wernicke's encephalopathy (WE) is an acute neuropsychiatric syndrome caused by thiamine deficiency and is associated with significant morbidity and mortality ${ }^{1}$. WE is a rare condition, characterized by altered mental status, as well as ocular, walking, and balance abnormalities. Although WE usually results from chronic alcohol dependence, nonalcoholic causes are reported in $20 \%$ to $50 \%$ of patients. WE rarely develops in patients with cancer. ${ }^{2,3}$ There have been few case reports of WE in patients with malignant lymphomas ${ }^{3}$. The occur- rence of HSCT-related WE is also rare, mainly in autologous HSCT ${ }^{4}$. WE occurrence is associated with drug use, prolonged Total Parenteral Nutrition (TPN), vomiting, and malnutrition. ${ }^{3,4}$

A 36-year-old female, married, small farmer, with no history of alcoholism was diagnosed with a bulky inguinal diffuse large B-cell non-Hodgkin lymphoma IIIB - $(10 \mathrm{~cm})$. She was admitted to the University Hospital to undergo an autologous HSCT. The mobilization was performed with Granulocyte Colony-Stimulating Factor, and 2.5 x $10^{6} \mathrm{CD} 34$ cells $/ \mathrm{kg}$ 
were collected. The conditioning regimen for autologous TCTH was BEAC (BCNU, etoposide, cytarabine, cyclophosphamide). The infusion of hematopoietic stem cells was performed, and on $\mathrm{D}+2$, she had a recurrence of fever, abdominal pain, and vomiting. On D+3 the patient's overall condition was worse, with contraction of diuresis, hypotension, and abdominal distension. Volume expansion with saline solution and vasoactive drugs were performed. On $\mathrm{D}+4$, a large amount of gastric residue was observed after nasogastric intubation. On the $\mathrm{D}+5$ there was an improvement of the patient's overall condition with weaning of the vasoactive drug and a gastric residue (GR) of $3650 \mathrm{~mL}$, followed by $1950 \mathrm{~mL}$ on $\mathrm{D}+6$, and $2,150 \mathrm{~mL}$ on $\mathrm{D}+7$. Abdominal pain and abdominal distension persisted, and TPN was introduced on $\mathrm{D}+7$. On D+12 the nasogastric tube (NT) was closed, and a restricted liquid diet was allowed. She had difficulty in accepting the oral diet, and the NT was maintained. On $\mathrm{D}+18$, recurrence of fever was observed, and the patient still had abdominal pain and distension and difficulty in walking. On D+22, weaning from TPN was performed, and there was an improvement in the abdominal condition.

Neutrophil grafting occurred only on D+23. On $\mathrm{D}+24$ the patient partially tolerated oral diet but still required blood transfusions. She developed apathy and drowsiness. On D+30, a new febrile peak was observed, with the reintroduction of Meropenem. A clinical scenario characterized by sensorineural fluctuation, the absence of focal signs, difficulty in ambulation and horizontal nystagmus was observed. The electroencephalogram (EEG) did not show any specific findings. On D+35 cerebrospinal fluid cytology revealed negative findings for malignancy. There was no evidence of central nervous system infection. PCR tests for herpes, dengue, and chikungunya fever were negative. Magnetic resonance imaging (MRI) of the brain showed increased signal in the fluid-attenuated inversion recovery (FLAIR) sequences around the Sylvian aqueduct and in the medial parts of both the thalamus and mammillary bodies (Figures 1a and 1b). Encephalitis Protocol (Meropenem, vancomycin, sulbactam sodium / ampicillin sodium, acyclovir) was empirically introduced. Thiamine replacement was initiated with $1500 \mathrm{mg}$ IV for 3 days, followed by $900 \mathrm{mg} /$ day. Improvement in the level of consciousness and nystagmus was quickly observed, while she persisted with temporal/spatial disorientation and recent amnesia. She was afebrile at discharge with hematologic recovery. The patient continued with progressive improvement of disorientation and amnesia but had pain in the lower limbs (neuropathy). During the follow-up, neurological changes and oral ingestion gradually improved.

Neurologic complications are frequently observed in patients during HSCT, being reported in 30\% to 39\% of cases $^{5}$. These complications may be infectious, cerebrovascular, toxic, immuno-mediated or metabolic $^{5}$. The complications may be due to drugs, thiamine deficiency, among others. Wernicke's encephalopathy (WE) is an acute neuropsychiatric syndrome

FIGURE 1. AXIAL FLAIR IMAGES OF THE BRAIN DEMONSTRATING AREAS OF SIGNAL CHANGE CHARACTERISTIC OF WERNICKE'S ENCEPHALOPATHY: IN (A), SYMMETRIC HYPERINTENSITIES IN THE MAMILLARY BODIES (ARROW) AND PERIAQUEDUCTAL GRAY MATTER; IN (B), SYMMETRIC HYPERINTENSITIES IN THE MEDIAL REGIONS OF THE THALAMUS.

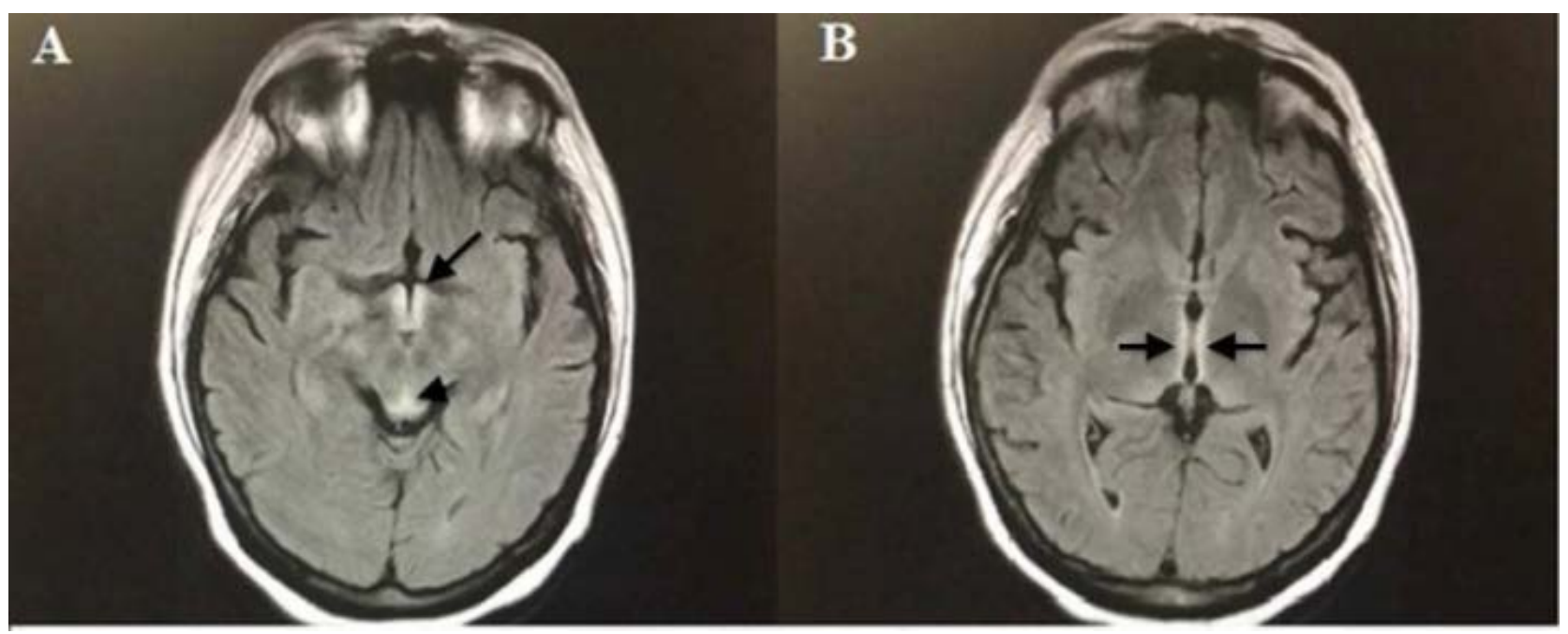


caused by thiamine deficiency that causes mental alterations, ocular, and balance abnormalities. Reports of WE cases in the literature associated with HSCT, mainly allogeneic transplantations, have been poorly described. Among the indicators of predisposition to WE in HSCT is prolonged total parenteral nutrition (TPN), since the latter is thiamine-deficient. ${ }^{5,6}$ Some authors have considered the prolonged use of TPN as the main risk factor for HSCT associated with Wernicke's Encephalopathy, but the duration of TPN required for the disease to manifest is unknown. ${ }^{7}$ Patients receiving long-term TPN, and intravenous solutions require higher amounts of thiamine to metabolize carbohydrate intake, which may rapidly consume thiamine stocks. ${ }^{3}$ Studies show that a state of depletion can develop within 18-20 days in patients receiving a strict diet without thiamine. ${ }^{5}$ Most of the reports concluded that prolonged TPN was the primary risk factor for HSCT-associated WE. ${ }^{4}$

Our patient with NHL underwent an autologous HSCT. Busulfan is not used in the conditioning regimen. She received TPN for approximately 2 weeks associated with episodes of vomiting. TPN includes multivitamin and mineral supplementation. The patient received prolonged TPN without thiamine, and WE symptoms appeared on day +35 . Wernicke's encephalopathy was diagnosed based on the history of consistent use of TPN and CNS symptoms with

\section{REFERENCES}

1. Turner JE, Alley JG, Sharpless NE. Medical problems in patients with malignancy: case 2. Wernicke's encephalopathy: an unusual acute neurologic complication of lymphoma and its therapy. J Clin Oncol 2004; 22:4020-4022.

2. Sechi G, Serra A. Wernicke's encephalopathy: new clinical settings and recent advances in diagnosis and management. Lancet Neurol 2007; 6:442-455.

3. Jung ES, Kwon O, Lee SH. Wernicke's encephalopathy in advanced gastric cancer. Cancer Res Treat 2010; 42:77-81.

4. Majolino I, Caponetto A, Scimé R, Vasta S, Fabbiano F, Caronia F. Wernicke-like encephalopathy after autologous bone marrow transplantation. Haematologica 1990; 75: 282.

5. Baek JH, Sohn SK, Kim DH, Kim JG, Lee HW, Park SP, et al. Wernicke's symmetrical T2/FLAIR hypersignal in the hypothalamic region, mammillary bodies and walls of the $3^{\text {rd }}$ ventricle, medial region of the thalamus (Figure 1), although the level of thiamine was not assessed.

Several drugs routinely used in HSCT are associated with neurological abnormalities, including busulfan $^{4}$ cyclosporine $\mathrm{A}^{5}$ and tacrolimus. ${ }^{6}$ We must consider that patients with HSCT are at high risk of acute encephalopathy due to chronic malnutrition, nausea induced by chemotherapy and vomiting ${ }^{8}$, by neurological alterations, including disorientation, mental state alteration, visual disturbances, and coma. ${ }^{5}$ However, it is yet unclear whether the use of these drugs during HSCT are risk factors for triggering such complications.

The consensus of the European Federation of Neurological Societies (EFNS) is that whole-blood thiamine (vitamin B1) measurement should be performed immediately prior to the administration of thiamine to confirm suspected or manifested WE, and MRI should be used to support the diagnosis. ${ }^{9}$ We conclude that WE should be considered in patients submitted to autologous HSCT associated with prolonged use of TPN and malnutrition.

\section{CONFLICTS OF INTEREST}

The authors declare having no conflict of interest. encephalopathy after allogeneic stem cell transplantation. Bone Marrow Transplant 2005; 35: 829-830.

6. LF Bleggi-Torres, BC de Medeiros, VSA Ogasawara, G Loddo, JZanis Neto, R Pasquini, et al. latrogenic Wernicke's encephalopathy in allogeneic bone marrow transplantation: a study of eight cases. Bone Marrow Transplant 1997; 20:391-395

7. Sklar EM. Post-transplant neurotoxicity: what role do calcineurin inhibitors actually play? AJNR. Am J Neuroradiol 2006; 27:1602-1603.

8. Kuo SH, Debnam JM, Fuller GN, de Groot J. Wernicke's encephalopathy: an underrecognized and reversible cause of confusional state in cancer patients. Oncology 2009; 76:10-18.

9. Solmaz S. Wernicke's Encephalopathy After Hematopoietic Stem Cell Transplantation. Turk J Hematol 2015; 32:367-370. 